\title{
DEL AULA PRESENCIAL A LA REMOTA Y DE REGRESO: LA ENSENANZA EN SITUACIONES DE PANDEMIA E INCERTIDUMBRE
}

\author{
FROM THE FACE-TO-FACE CLASSROOM TO THE \\ DIGITAL ENVIRONMENTS AND BACK: \\ TEACHING IN SITUATIONS OF PANDEMIC \\ AND UNCERTAINTY
}

Karen González-Fernández ${ }^{1}$

https://orcid.org/0000-0003-0773-7614

Recibido: septiembre 22, 2020 - Aceptado: octubre 27, 2020

\section{RESUMEN}

La pandemia provocada por el virus del COVID 19 trajo cambios importantes para muchos modelos educativos. La enseñanza presencial se convirtió, de manera brusca e inesperada, en enseñanza en entornos digitales remotos. El objetivo de este artículo es presentar una discusión sobre uno de los mayores retos que los sistemas educativos enfrentan en contextos como el presente: la incertidumbre generada por el cambio de la enseñanza presencial a la enseñanza remota y viceversa. Para lograr el objetivo: 1) muestro de qué manera el cambio de la enseñanza presencial a la remota provoca un problema al nivel de la planeación y el diseño de los cursos, y un reto específico para la labor docente, y 2) ofrezco el esbozo de una propuesta para enfrentar este tipo de situaciones, recurriendo al modelo de diseño instruccional ADDIE y considerando algunas competencias docentes específicas. Finalmente propongo unas reflexiones sobre algunos retos que la pandemia ha generado para la pedagogía en general y los diversos modelos educativos en particular.

Palabras clave: enseñanza presencial, enseñanza remota y a distancia, incertidumbre, pandemia.

1 Doctora en Filosofía de la Ciencia, UNAM. Profesora-Investigadora, Universidad Panamericana, México. Miembro de la Academia Mexicana de Lógica. kgonzale@up.edu.mx 


\begin{abstract}
The pandemic caused by the SARS-CoV-2 virus brought important changes for many educational models. Face-to-face classes had to move to virtual environments abruptly and unexpectedly. The objective of this article is to present a discussion about one of the greatest challenges that educational systems have to confront in contexts such as the present: the uncertainty generated by the change from face-to-face to remote teaching and vice-versa.
\end{abstract}

To achieve the objective, 1) I show how the change from face-to-face to remote classes in digital environments causes a problem at the level of the planning and design of courses and a specific challenges for teaching; and 2) I offer the outline of a proposal to face this type of situation using the ADDIE model of instructional design and considering some specific teaching competences. Finally, I offer some final reflections on the challenges that the pandemic has generated for pedagogy and educational systems.

Keywords: Face-to-face Teaching, Remote Teaching, Uncertainty, Pandemic.

\title{
1. INTRODUCCIÓN²
}

El año 2020 será recordado sin duda por la pandemia del COVID 19, que ha generado importantes modificaciones en múltiples áreas de la vida cotidiana de las personas y las instituciones, y organizaciones de todo tipo.

Uno de los sectores más afectado por este fenómeno es la enseñanza presencial escolarizada. Las escuelas han debido cerrar sus puertas físicamente, y en la mayor parte de los casos y en todos los niveles educativos, han trasladado su labor a los entornos digitales con clases en línea ofrecidas de manera remota (donde los docentes y estudiantes no comparten un espacio físico), brindadas a través de diversos servicios y plataformas disponibles en Internet.

Los modelos de educación remota o a distancia no son nuevos en el año 2020, sin embargo, para quienes estaban acostumbrados a trabajar en entornos de enseñanza presencial, ha significado un gran reto trasladar sus cursos exclusivamente al modelo de enseñanza remota en entornos digitales.

En este artículo mostraré que uno de los mayores retos a los que se enfrenta la docencia en el contexto de la presente pandemia no es, principalmente, el cambio a la enseñanza remota, sino que, en general, no contamos con las suficientes herramientas para diseñar un curso exitoso -independientemente de si se realiza de manera presencial o a distancia- y que permita pasar de un modelo a otro en cualquier momento no previsto de antemano.

Al no saberse cuándo se tendrán clases de manera presencial, y que eso podría ocurrir en cualquier momento, sostengo que el factor de incertidumbre que genera la pandemia ha

2 Agradezco las observaciones de los dictaminadores anónimos, y el intercambio de ideas con Renato Huarte Cuéllar y Tatiana Lozano Ortega, que fueron fundamentales para la elaboración de la versión final de este artículo. 
causado un problema para el que los docentes no estaban preparados. En este artículo: 1) discutiré la manera en que aparece la incertidumbre en las distintas áreas relacionadas con la planeación y la ejecución de los cursos presenciales que se han visto afectados por la pandemia; 2) esbozaré una propuesta para enfrentar esta problemática desde la planeación y el diseño de los cursos, centrada en el modelo ADDIE y en competencias docentes para enfrentar la incertidumbre en la planeación, y 3) ofreceré algunas reflexiones finales acerca de los retos que se le presentan a los modelos educativos presenciales a partir de la consideración de los cambios generados por la pandemia.

El objetivo de este artículo es mostrar que hace falta generar un modelo de enseñanza que permita pasar de lo presencial a lo remoto en cualquier momento y que este es un reto interesante para la pedagogía, disciplina que debe encontrar la manera de incluir factores de incertidumbre -como los generados en la pandemia- en sus propuestas de modelos de planeación y desarrollo de cursos formales. Además, espero que estas reflexiones sean útiles para los docentes que han debido enfrentar esta situación inédita en su labor cotidiana y que, durante y después de la pandemia, deberán replantear sus prácticas y metodologías.

\section{INCERTIDUMBRE: UNO DE LOS PRINCIPALES RETOS PARA LA PLANEACIÓN Y DISEÑO DE CURSOS EN EL CONTEXTO DE LA PANDEMIA}

Entre los cambios que se han debido introducir por la pandemia, se encuentra la modificación radical de la educación escolarizada en formato presencial. En México, a partir del 23 de marzo de 2020 - fecha en que la Secretaría de Educación Pública determinó que todas las escuelas cancelarían sus actividades presenciales ${ }^{3}-$, los ciclos escolares que finalizaban cerca del verano se interrumpieron en sus labores cotidianas. En un inicio se preveía que esta interrupción escolar duraría hasta el mes de abril; sin embargo, la situación de la pandemia fue escalando y hasta el momento (noviembre de 2020) no se tiene claro cuándo podrán reactivarse las clases presenciales en el país. Esta situación obligó a terminar las actividades escolares del primer semestre de 2020 de manera remota, y a comenzar las actividades del segundo semestre de este año también en este formato.

Muchas escuelas de todos los niveles educativos trasladaron sus actividades al formato de enseñanza remota en entornos digitales desde el inicio de la suspensión de clases presenciales. El segundo semestre de 2020 obligó a las instituciones educativas, incluyendo las públicas, a replantear todos sus procesos de enseñanza-aprendizaje y generar por lo menos algunas actividades en términos de los modelos de educación remota, en donde se volvió imperativo programar las clases a través de medios audiovisuales, como la televisión y el Internet.

Estos cambios vinieron aparejados de varios problemas: 1) el poco tiempo para realizar el cambio al formato remoto, con el uso generalizado de los entornos y las herramientas digitales, y 2) la inseguridad acerca de cuándo terminará la situación «extraordinaria» y se volverá a impartir clases en entornos presenciales. 
A pesar de las dificultades para adecuarse a las clases remotas -literalmente de la noche a la mañana-, considero que la incertidumbre sobre el formato de las clases (presenciales o a distancia) es un problema más grave y que no se ha tenido lo suficientemente en cuenta.

En este caso específico, la incertidumbre viene determinada por la pandemia: la manera en que se propaga y el éxito que se tiene o no al combatirla. En estos momentos, la carrera por generar una vacuna tiene a los científicos trabajando a marchas forzadas y a la población en general esperando a que lleguen los anuncios de que todas las actividades se desarrollarán presencialmente; entre ellas, la educación escolarizada, una de las últimas que podrá volver a realizarse de este modo.

Los centros educativos de todos los niveles han debido realizar sus actividades en los últimos meses con este telón de fondo: no se sabe cuándo se regresará a las instalaciones físicas y es posible que durante algún período de tiempo (que tampoco se sabe cuánto durará) se trabajará en un sistema «híbrido» en el que algunas clases se impartirán presencialmente y otras remotamente. Tampoco es seguro que llegue a presentarse este período «híbrido» de clases. Esta es la situación que resulta realmente inédita: nunca se había planteado un escenario de planeación y diseño de cursos en el que debiera lidiarse con este nivel y esta presentación de la incertidumbre. Esto es lo que vuelve complejo el panorama presente y lo que representa un reto mayor para la comprensión de las circunstancias en general, y para la planeación y ejecución de las clases, antes presenciales y ahora obligadamente remotas, en el contexto actual.

Desde que se establecieron las políticas de encierro, profesores y estudiantes que trabajaban en el sistema presencial tuvieron que conocer y acostumbrarse a funcionar a distancia. Esto supuso un reto importante, tanto para docentes como para estudiantes. En muchas instituciones se generaron cursos y sistemas de capacitación para los profesores con el objetivo de enfrentar esta situación extraordinaria ${ }^{4} \mathrm{y}$ fue obligatorio actualizarse en las tecnologías digitales más afines a la enseñanza remota, al mismo tiempo que el fenómeno de la pandemia modificaba todas las áreas de la vida cotidiana de las personas y las obligaba a tomar medidas sanitarias para evitar la propagación del virus. Se exigió a los profesores y a los estudiantes a que terminaran el ciclo escolar, iniciado de manera presencial, en un «modelo remoto de emergencia» (cfr. Hodges et al., 2020) en el que, además, se buscaba no perder la calidad de la educación y del intercambio intelectual entre alumnos y docentes. Esto no se logró de manera exitosa en todos los casos y hubo muchas variaciones en la manera en la que cada profesor y cada estudiante vivió esta experiencia ${ }^{5}$.

Para quienes ya trabajaban como profesores en modelos en línea o a distancia, o que ya empleaban plataformas educativas en Internet, el cambio del entorno presencial al remoto resultó un poco más sencillo, a diferencia de quienes no habían ocupado antes estas modalidades educativas o tecnologías disponibles en Internet. Y por este motivo, muchos de quienes han generado los cursos y las capacitaciones para la enseñanza en entornos digitales son quienes trabajaban desde antes en las áreas de educación en línea y a distancia de las diferentes instituciones.

4 Véase, por ejemplo, el proyecto Apolo 21 de la Universidad Panamericana https://www.up.edu.mx/es/noticias/37869/apolo-21-acompana-docentes-en-desarrollo-online y la oferta de cursos para modelos de enseñanza en línea y a distancia que ofrece la UNAM: $\underline{\text { https: / / cuaieed.unam.mx/ }}$

5 Una interesante recopilación de diferentes experiencias de enseñanza durante la pandemia por Covid 19 se encuentra en Jandrić, et al. (2020). 
Los cursos de capacitación que suelen ofrecerse a los docentes cubren una amplia gama de cuestiones relacionadas con la enseñanza en línea: cuáles son las principales herramientas tecnológicas con las que se cuenta para los ámbitos educativos, cómo mantener la atención de los estudiantes en entornos digitales, cómo generar recursos educativos en medios audio-visuales como videos, por ejemplo, o cuáles son las mejores metodologías para enseñar a distancia ${ }^{6}$.

Sin embargo, es muy difícil que en las diversas capacitaciones se considere la particularidad de la situación en la que nos encontramos y que ya he mencionado anteriormente: la incertidumbre que implica no saber si los cursos serán completamente remotos, completamente presenciales o bien «híbridos». Incluso aunque se pueda prever que al menos cursos inmediatamente próximos serán completamente remotos, no hay aún una garantía de eso. Y en términos generales no se piensa que el cambio al modelo remoto será permanente o altamente significativo, cuando en realidad podría llegar a serlo.

Esto provoca problemas de diversa índole: quienes trabajan en modelos en línea o a distancia suelen tener paradigmas en los que se considera que la carga de la educación está en el alumno, quien es muy independiente y se vuelve responsable de su aprendizaje de una manera que, por lo menos, no suele presentarse en entornos presenciales. Es muy común tener la idea de que, en estos modelos, el estudiante es prácticamente autodidacta y que la labor del profesor o asesor (si es que existe esta figura) es principalmente de acompañamiento y evaluación del aprendizaje obtenido (cfr. Goodyear, Salmon, Spector, Steeples y Tickner, 2001). En consecuencia, también la duración y cantidad de clases o asesorías remotas es muy diferente a la que suele darse en los entornos presenciales; generalmente existen menos sesiones "síncronas», en donde se encuentren al mismo tiempo estudiantes y profesores y realicen un intercambio comunicativo directo; y surgen más actividades «asíncronas», que el estudiante puede revisar o llevar a cabo en el momento en que a él le convenga. No es que exista una relación necesaria entre la enseñanza remota con las actividades asíncronas y los entornos presenciales con las actividades síncronas, pero considero que plantear la problemática desde esta perspectiva puede ser un buen punto de partida para la propuesta que plantearé más adelante.

En el caso de los entornos de clases presenciales que se transformaron en clases remotas debido a la pandemia, la manera de entender la relación profesor-estudiante y de asignar los tiempos a las distintas actividades no suele estar bien especificada. Es decir, se asume que la relación digital entre profesor y estudiante es prácticamente igual a la relación presencial, o que las diferencias no son significativas; y en muchos casos se asigna el mismo número de clases y de duración en la modalidad remota a las que se tenía en el modelo presencial. Este esquema favorece la hipotética situación de que se volverá a las clases presenciales en cualquier momento, pero no es claro que sea la mejor estrategia para favorecer el aprendizaje y la enseñanza en el caso de que las clases deban desarrollarse de manera remota. Algunas instituciones han dado flexibilidad para que los profesores realicen sus clases con diferentes metodologías, y con cantidades distintas de actividades síncronas y asíncronas; pero esto no sucede siempre y, además, los profesores pueden no tener clara la diferencia y las problemáticas de los procesos de atención y concentración en los entornos digitales versus los mismos procesos en entornos presenciales, por lo cual podría resultar mermada la calidad de los cursos al no diferenciar cuáles son los cambios significativos a realizar para impartir clases de manera remota en entornos digitales. 
Por otro lado, tampoco debe pensarse que basta con familiarizarse por completo con la modalidad de enseñanza en línea y con volverse experto en el uso de las tecnologías digitales y el conocimiento de los procesos de aprendizaje en estos entornos, pues como he mostrado, el problema es que eventualmente se volverá a las clases presenciales y esto requerirá, seguramente, hacer nuevos ajustes; y está siempre de fondo la problemática de que no se sabe en qué momento sucederá eso.

Esta situación inédita generada por la pandemia presenta un reto altamente significativo para la pedagogía, disciplina que debe buscar generar propuestas de planeación de cursos y de estrategias de enseñanza-aprendizaje para enfrentar escenarios con altos factores de incertidumbre como los descritos aquí. Dado que se prevé que es muy probable que empiecen a repetirse más constantemente escenarios como el actual, es importante generar modelos que permitan integrar tanto entornos presenciales como remotos y que ofrezcan la posibilidad de pasar de unos a otros, en cualquier momento.

Considero que una guía mínima para que los docentes enfrenten de mejor manera situaciones de emergencia, como la generada por la pandemia del Covid 19, debiera incluir:

1) Ofrecer modelos para diseñar y planear los cursos que sean lo suficientemente flexibles para introducir los cambios que hagan falta cuando se pase de un entorno presencial a uno remoto y viceversa.

2) Brindar capacitaciones y recursos para que los docentes conozcan y se familiaricen con el uso de las herramientas tecnológicas a su disposición, y se pueda profundizar en el conocimiento de las que sean más adecuadas a cada disciplina y modalidad (remota o presencial).

3) Procurar herramientas a los profesores para generar material y estrategias didácticas, que puedan usarse tanto de manera síncrona como asíncrona ${ }^{7}$.

4) Propiciar el desarrollo de competencias que impliquen no solo conocer a profundidad las características de los modelos de educación a distancia, sino también contar con herramientas para combinar esta modalidad con la presencial cuando haga falta, con el objetivo final de que -independientemente de cualquier cambio en el entorno y del momento en que este suceda- los objetivos del curso puedan cumplirse.

A continuación, presentaré una breve propuesta de cómo podría enfrentarse el problema planteado en el punto 1, referente al diseño de los cursos, y posteriormente me concentraré en el punto 4, concerniente a las competencias que los docentes deberían tener en cuenta, pensando sobre todo en el factor de incertidumbre relativo a los cambios intermitentes de modalidades presenciales a modalidades a distancia. 
No es mi objetivo desarrollar exhaustivamente ambas propuestas, sino solo ofrecer algunos elementos útiles para los docentes que se enfrentan a la situación de impartir clases en contextos de incertidumbre como el descrito aquí, y por otro lado, incoar algunas ideas que podrían ser útiles para generar propuestas pedagógicas mucho más estructuradas.

\section{EL MODELO ADDIE COMO HERRAMIENTA PARA DISEÑAR CURSOS EN CONTEXTOS DE INCERTIDUMBRE}

Para introducir de mejor manera el elemento de incertidumbre en el diseño, la planeación y ejecución de cualquier clase, es importante contar con un modelo pedagógico que permita introducir variaciones en cualquier etapa del proceso educativo, sin que esto requiera cambios significativos o mucho tiempo para modificar las planeaciones. Teniendo esto en cuenta, ofreceré una propuesta basada en el modelo ADDIE para lograr este objetivo.

El modelo ADDIE es una propuesta de diseño instruccional $l^{8}$ planteado, originalmente, para desarrollar cursos a distancia en los que se buscaba que el alumno fuera adquiriendo autonomía y en los que las actividades propuestas se realizaban con herramientas digitales; sin embargo, es un modelo que puede trasladarse también a entornos presenciales. ADDIE surgió a mediados de la década de los 70, y desde entonces, se han propuesto actualizaciones al mismo en diversas ocasiones (cfr. Morales-González, Edel-Navarro y Aguirre-Aguilar, 2014: 35).

El nombre de ADDIE se obtiene del acrónimo en inglés de las diferentes partes que conforman a este modelo: análisis, diseño, desarrollo, implementación y evaluación. Describiré brevemente cada etapa:

1) Análisis: se evalúa quiénes serán los estudiantes, los contenidos a enseñar y el entorno en el que se desenvolverá el curso.

2) Diseño: se prepara el contenido del curso a impartir, se plantea el enfoque didáctico con el que se impartirá el curso, se generan actividades e instrumentos de evaluación, se preparan las actividades de aprendizaje a llevar a cabo y se definen los recursos que se utilizarán.

3) Desarrollo: se realiza la implementación de los recursos que se utilizarán en las plataformas educativas o las páginas web; se elaboran y prueban.

4) Implementación: se da la interacción con los alumnos y se realizan propiamente las actividades de aprendizaje.

5) Evaluación: se valoran los resultados obtenidos en el curso, así como los procesos y los recursos de enseñanza-aprendizaje. Además se puede evaluar cada fase, con lo cual se pueden hacer ajustes cuando sea necesario?.

8 El «diseño instruccional» se entiende, según Bruner (1969), como la disciplina que se ocupa de «la planeación, la preparación y el diseño de los recursos y ambientes necesarios para que se lleve a cabo el aprendizaje» (citado por Belloch, p. 2). Para analizar otras maneras de entender el diseño instruccional, cfr. Belloch, 2011, p. 2.

9 El modelo ADDIE se emplea actualmente en entornos empresariales y educativos. Para una descripción más amplia de lo que es el desarrollo instruccional, modelos como ADDIE y otras propuestas puede consultarse a Morales-González, et al., 2014; Esquivel, 2014; Góngora Parra y Martínez Leyet, 2012; Jardines, 2011; Londoño, 2011, y Chiappe, 2008. 
Como he mencionado, este modelo surgió para propuestas de educación digital y en su origen está dirigido a la realización de tareas y actividades a través de plataformas como Moodle o Edmodo, por ejemplo. Esto favorece su aplicación en el contexto presente; sin embargo, mi propuesta es entender el modelo de manera más amplia para incluir, si se llega a dar el caso, actividades a realizar en entornos presenciales, con las particularidades que implique la vuelta a las aulas físicas.

Mi propuesta es que la planeación se realice para un modelo de enseñanza remota, pero con la consideración -desde el inicio- de una posible vuelta a las aulas presenciales; en la medida de lo posible, el curso debe diseñarse de manera que todas las actividades propuestas para el entorno digital, se consideren para la realización completa del curso, independientemente de las actividades que puedan ser presenciales. Sin embargo, deben incluirse actividades presenciales en caso de regresar a las aulas en cualquier momento, antes de que termine el curso que se esté impartiendo.

Generar la planeación de todo un curso a partir de un modelo como ADDIE, puede ofrecer a los docentes la flexibilidad suficiente para modificar la planeación y ejecución del curso cuando haga falta, sin perder de vista los objetivos principales del proceso de enseñanza-aprendizaje y asegurando la consecución exitosa de este último, independientemente de las circunstancias - presenciales o a distancia- generadas sobre la marcha.

El modelo ADDIE es uno de los más adecuados para el manejo del factor de incertidumbre del cambio intermitente de los modelos de enseñanza presencial a los modelos de enseñanza remota, por las siguientes razones:

1) Incluye de forma natural las herramientas digitales, imprescindibles para la enseñanza remota, pues fue generado precisamente para trabajar con ellas. Dado que la pandemia ha obligado a recurrir a este tipo de herramientas, resulta adecuado trabajar con un modelo que las tiene en cuenta desde el inicio.

2) En él, todos los elementos se supeditan a los objetivos de enseñanza-aprendizaje; esto implica que las herramientas tecnológicas a usar estarán al servicio de los objetivos buscados, lo que privilegiará el aprendizaje independientemente del formato (presencial o remoto) en el que se impartan las clases.

3) La etapa del «análisis» resulta fundamental para enfrentar el problema de la incertidumbre que propongo como central en este artículo. Dado que esta es una etapa previa al diseño del curso, permite tener en cuenta las particularidades propias de cada situación, de modo que favorece los posibles cambios en los entornos y procesos de enseñanza-aprendizaje que podrían generarse durante el curso mismo si es que, por ejemplo, se inicia un curso de manera remota y en algún momento debe cambiarse el formato a un modelo presencial. La inclusión de esta etapa de «análisis» en el modelo, permite prever las diversas posibilidades y contribuye a un flexible diseño del curso en caso de que haga falta.

4) En la etapa de «diseño» pueden proponerse varios tipos de actividades que facilitarán la labor docente, si existiera un cambio de formato sobre la marcha: a) actividades digitales que funcionen tanto en la modalidad presencial como en la modalidad remota; b) actividades adecuadas solo para la modalidad remota; c) actividades adecuadas solo para la modalidad presencial.

El docente podría diseñar desde el inicio del curso los tres tipos de actividades, o podría privilegiar la preparación de algún tipo, previendo cuál es la modalidad en la que más 
probablemente se desarrollará su curso, pero teniendo claro también que las circunstancias podrían cambiar en cualquier momento y que, de ser así, debería dedicar un tiempo extra a desarrollar las actividades de la otra modalidad cuando haga falta.

Con lo dicho hasta aquí he mostrado que planear un curso de acuerdo con el modelo ADDIE podría ayudar a tener en cuenta el elemento de incertidumbre relativo a los posibles cambios de formato del curso (presencial o remoto), lo cual evitará que una modificación brusca del mismo sea considerada, necesariamente, un factor de «emergencia». Así, aunque se cambie el formato, si estábamos preparados para ello y no perdemos de vista los objetivos, el ajustar las actividades -de modalidad presencial a remota o viceversa-, no tendría por qué afectar de manera negativa los procesos de enseñanza-aprendizaje y el logro de los objetivos del curso.

De cualquier modo, diseñar un curso con las características «híbridas» planteadas no es sencillo, e implementarlo tampoco. Entre otras cosas, se requiere que los docentes adquieran $\mathrm{o}$, si ya las tienen, ejecuten hábilmente ciertas competencias que posiblemente no se habían considerado tan relevantes como ahora parecen serlo, debido al contexto de la pandemia. Por este motivo, el siguiente apartado reflexiona sobre cuáles son algunas de las competencias docentes que es deseable desarrollar en contextos de incertidumbre como el presente.

\section{COMPETENCIAS DOCENTES EN CONTEXTOS DE INCERTIDUMBRE}

\subsection{Enseñanza presencial versus enseñanza remota}

Por todo lo dicho, los docentes deben prepararse para enfrentar escenarios con cursos en presentación remota y otros en modelo híbrido: parte a distancia y parte presencial. Además, hay que tener en cuenta que en los sistemas educativos oficialmente presenciales aunque todos los cursos de uno o varios semestres se realicen, por el momento, en presentación remota, se espera que pueda regresarse a los entornos presenciales en algún momento; de modo que al final, la enseñanza a distancia será solo una pequeña parte de la totalidad de la formación de los estudiantes adscritos a estos programas.

Esto implica que la manera ideal de enfrentar esta situación no debería ser pedir a los profesores de modalidades de enseñanza presencial que cambien radicalmente su metodología y forma de trabajar a modelos diseñados únicamente para enseñanza remota. En todo caso, hay que tratar de adquirir y conservar lo mejor de ambos sistemas, hasta donde sea posible, y cada profesor decida el estilo que dará a sus cursos en específico y a sus prácticas docentes, idealmente tratando de que estos elementos funcionen adecuadamente tanto en versión remota como presencial, o si esto no es del todo posible, buscando una transición armónica de un modelo al otro.

Para lograrlo, es importante tener en cuenta las principales características de la enseñanza presencial y de la enseñanza remota.

En consecuencia, presento a continuación un listado con las principales características que se atribuyen a cada tipo de modelo para, posteriormente, discutir sus semejanzas y diferencias. La caracterización que presento es general y no cubre todas las maneras 
posibles de implementar cursos y realizar evaluaciones; pero quiero presentar algunas de las prácticas tradicionales que suelen realizarse en ambos entornos y que pueden plantear problemáticas significativas a la hora de hacer transiciones de uno a otro.

\section{a) Características de la enseñanza en modelos presenciales}

- Se diseña cada curso estableciendo con anticipación su objetivo, el material con el que se estudiará, las actividades que realizarán los estudiantes y los métodos e instrumentos de evaluación.

- Se explican contenidos teóricos de manera clara, precisa y sintética, usando herramientas como pizarrón, exposición y lectura de textos. En ocasiones, los docentes se apoyan de herramientas digitales como presentaciones audiovisuales $u$ otras; pero el empleo de estas tecnologías surge, en muchos casos, en contextos síncronos, en donde existe una interacción entre el profesor y el estudiante, en el mismo espacio y al mismo tiempo.

- Se espera que el docente adapte su programa y sus actividades según la retroalimentación inmediata o las cuestiones no previstas.

- Los profesores resuelven dudas y problemas de manera síncrona.

- Se evalúa el desempeño de los alumnos, lo cual se da también en muchos casos de manera síncrona, en sesiones en donde el profesor y los estudiantes se encuentran en el mismo espacio, y el profesor puede controlar si el estudiante consulta material o no, y qué material puede consultar.

\section{b) Características de la enseñanza remota o a distancia}

- Se diseña el curso estableciendo con anticipación los objetivos del mismo, el material con el que se estudiará, las actividades que realizarán los estudiantes y los métodos e instrumentos de evaluación.

- Se explican contenidos teóricos, de manera clara, precisa y sintética usando herramientas digitales como videos, pizarrones digitales o presentaciones audiovisuales. Generalmente las sesiones síncronas -mediante alguna plataforma de Internet- son de menor duración y más espaciadas.

- Se espera que los estudiantes sean más independientes, por lo que el papel del profesor es más de motivador que de facilitador de contenidos.

- El profesor o los diseñadores del curso producen material para ser consultado de manera asíncrona, como videos, infografías, mapas conceptuales o actividades mediadas por las plataformas que, en algunos casos, pueden generar retroalimentación inmediata y automática.

- El profesor resuelve dudas y problemas, generalmente de manera asíncrona.

- Se evalúa el desempeño de los alumnos, en muchas ocasiones de manera asíncrona, y con actividades muy diferentes a las presentadas en los entornos presenciales. Muchas veces, la evaluación implica pedir al estudiante que responda preguntas con respuestas cerradas para las que la evaluación es automáticamente generada por la plataforma digital utilizada. 


\subsection{Sincronicidad y asincronicidad, ¿hasta dónde?}

Como se aprecia a partir de las listas anteriores, las principales semejanzas entre ambos modelos de enseñanza - presencial y remoto- se encuentran en que se espera que los docentes diseñen sus cursos con anticipación, ofrezcan explicaciones claras de sus materias (para lo cual deben ser expertos en el conocimiento del contenido de las mismas), propongan actividades que favorezcan el aprendizaje de los alumnos y evalúen adecuadamente a los mismos.

Si bien, estas similitudes parecen claras, no hay que perder de vista que la manera en que se realizarán las clases determinará en buena medida el tipo de recursos que los docentes eligen para sus cursos, actividades y métodos de evaluación; lo cual nos lleva a una gran diferencia entre las dos modalidades de enseñanza discutidas aquí y que se relaciona con un elemento de aparición recurrente en el listado anterior: la sincronicidad (asociada a modelos de enseñanza presencial) y la asincronicidad (ligada a modelos de enseñanza remota).

Considero que las decisiones que tomen los docentes sobre las actividades síncronas y asíncronas que realizarán en sus cursos, determinarán la manera en que se propondrán los procesos de diseño del curso. Mostraré cómo aparecen estos factores en un caso hipotético: supóngase que se impartirá un curso que iniciará de manera remota. Desde esta perspectiva, la planeación del curso, siguiendo el modelo ADDIE, sería:

1) En el análisis, habrá que tener en cuenta los recursos de los alumnos: ¿tienen acceso a Internet con algún dispositivo con cámara, por ejemplo? Si la mayoría de los alumnos no cuenta con esto o es de esperar que tendrá dificultades en este sentido, será mejor incluir una mayor cantidad de actividades asíncronas.

2) La cantidad de recursos asíncronos que se consideren, puede llegar a determinar también la duración y cantidad de sesiones síncronas en entornos virtuales que se tendrá con los alumnos, lo cual es un factor relevante para el momento del diseño del curso. Entre más recursos asíncronos se generen, la duración de las sesiones síncronas tenderá a ser menor, y viceversa.

3) En la etapa de desarrollo implicará un trabajo muy diferente el generar material asíncrono que material síncrono. No es lo mismo realizar un video o diseñarlo, y subir un cuestionario a una plataforma educativa, que preparar una explicación que se expondrá en persona a un grupo de estudiantes utilizando un pizarrón dentro de un aula. Este momento del modelo requiere practicar diferentes tipos de tecnologías y recursos del docente, dependiendo de cuál sea más adecuado para cada caso.

4) En la etapa de implementación, la principal diferencia está en lo que involucra -en términos comunicativos- que el profesor comparta el mismo tiempo y espacio con sus estudiantes... a que no lo haga. Cuando las sesiones son síncronas, surgen preguntas en vivo y la retroalimentación se da de inmediato; esto genera un entorno en donde las interacciones no están predeterminadas de antemano y los estudiantes tienen oportunidad de solucionar sus dudas en el momento en que se generan. En cambio, en el trabajo en modalidad asíncrona, el estudiante no tiene oportunidad de plantear dudas en el momento en que surgen, y el intercambio comunicativo entre profesor y estudiante se realiza a través de herramientas como el correo electrónico, por ejemplo.

5) Finalmente, como ya analizamos, la evaluación en el modelo ADDIE puede darse a muchos niveles, pero considerando solamente la situación de evaluar el conocimiento de los estudiantes, también se plantean diferencias importantes dependiendo de si la evaluación nace en contextos de sincronicidad que de asincronicidad. En el primer caso, será 
posible plantear evaluaciones en las que el profesor puede tener mucho control sobre la información que los estudiantes pueden consultar, mientras que en los contextos de asincronicidad esto no sucede.

En el caso hipotético que he planteado, si el profesor decidió incluir muchas actividades asíncronas en su planeación, una eventual vuelta a la modalidad de clases presenciales antes de lo esperado podría causarle dificultades, pues debería generar con poco tiempo las adecuadas a la modalidad de clases presenciales.

¿Cómo enfrentar este panorama tan poco determinado, de manera que el profesor planee su curso, desarrolle sus actividades y las lleve a cabo, sin que un cambio del modelo remoto al presencial pudiera representarle un problema mayor? Además, sería deseable contar con modelos de planeación y diseño de cursos adecuados para esto; la gran área de oportunidad para los profesores está en la adquisición de competencias que les ayuden a enfrentar estos escenarios. A continuación, presentaré algunas de las que considero más relevantes en un contexto como el estudiado aquí.

\subsection{Competencias docentes en contextos de incertidumbre sobre la modalidad presencial o remota}

Existen amplias investigaciones sobre las competencias que un docente debe poseer o desarrollar. Con la aparición de los modelos de enseñanza remota en entornos digitales, se generó un campo de estudio fructífero relativo a este apartado; existen múltiples artículos enumerando las principales habilidades que los docentes de este tipo de modelos deben poseer ${ }^{10}$. También se publica mucha literatura sobre las competencias docentes en general, y es interesante notar que hay menos trabajos sobre este tema para modelos presenciales, posiblemente esto se da por supuesto en los estudios sobre competencias docentes en general, pero valdría la pena cuestionarse mucho más sobre la diferencia entre los modelos de enseñanza presencial y a distancia, desde la perspectiva de las competencias docentes para cada caso $^{11}$.

A continuación, enlisto competencias que considero esenciales para enfrentar una situación como la de la pandemia de COVID 19, en la que bruscamente se tuvo que cambiar de clases presenciales a remotas, y posiblemente deberá también realizarse el movimiento a la inversa. En general, esta lista incluye habilidades para ambos modelos pero, sobre todo, me gustaría enfatizar que lo importante es prever lo que los cambios inesperados de un modelo a otro podrían generar y cuál podría ser una buena manera de estar preparado para esos cambios en caso de darse. No pretende ser una lista exhaustiva, sino solo una guía que ayude a los docentes a pensar en los problemas y las situaciones que podrían prevenir, la manera en que deberían proponer sus actividades y las capacitaciones que requerirían tomar.

- Apertura a capacitarse constantemente en el uso de las herramientas tecnológicas para la enseñanza: dado que los modelos de educación a distancia en entornos digitales son cada vez más una constante, los docentes acostumbrados a trabajar en

10 Cfr. Becerril, Sosa, Delgadillo y Torres (2015); Goodyear et al. (2011), solo por mencionar algunos.

11 Para analizar ambos tipos de competencias, puede consultarse a Gallego, 2007; Durán, 2015; Sotelo, Vales, García y Barrera, 2017. 
modelos presenciales deben estar sumamente abiertos a aprender qué herramientas tecnológicas existen y a incorporarlas en su labor cotidiana. Muchas de las herramientas tecnológicas pueden usarse también en cursos de carácter presencial; familiarizarse con ellas hará mucho más sencillas las transiciones de unos modelos a otros.

- Ejercitarse en el diseño de actividades tanto para entornos síncronos como asíncronos: como hemos visto, los modelos presenciales implican planear una gran cantidad de actividades síncronas, mientras que los modelos remotos requieren actividades asíncronas. El docente capaz de desarrollar ambos tipos de actividades para los mismos contenidos, tendrá menos problemas para generar nuevas actividades o modificar las que ya tenía preparadas cada vez que deba hacer un cambio brusco de un modelo a otro.

- Analizar cada actividad e instrumento de evaluación que se proponga, de modo que el docente tenga claro a qué modelo se adecua más: presencial, remoto o ambos.

- Organizar convenientemente el tiempo para desarrollar todos los elementos relativos al diseño del curso y a todas las etapas de desarrollo del mismo, en cualquier modalidad.

- Prever que si llegan a presentarse cambios bruscos de un modelo presencial a uno remoto o viceversa, seguramente se requerirá un esfuerzo y tiempo extra por parte del docente para adaptar el curso al cambio.

- Evaluar sobre la marcha las propias circunstancias antes de cada curso y al finalizar el mismo, detectar fortalezas y áreas de oportunidad; tratar de mejorar aún más las primeras y enfrentar las segundas. Si uno tiene más habilidades para las clases en el modelo presencial, debería fortalecer los aspectos que se le dificultan más en los modelos remotos y viceversa.

\section{REFLEXIONES FINALES}

La pandemia provocada por el virus del COVID 19 provocó un cambio radical en los sistemas educativos en un período de tiempo muy corto. Como mencioné en la introducción y ya señalaron Charles Hodges et al. (2020), no deberíamos pensar que de golpe empezamos a tener sistemas de enseñanza en línea bien establecidos, sino que más bien debimos adaptarnos a realizar una «enseñanza remota de emergencia». Si bien no se pueden prever todos los escenarios de incertidumbre a los que nos enfrentaremos, sí existen algunos en que las variables en juego están bastante bien definidas, por lo que no creo que debamos pensar que estamos condenados a repetir constantemente estas situaciones de emergencia.

En este artículo he mostrado que una de las principales diferencias entre los sistemas de enseñanza presencial y los de enseñanza remota radica en las actividades síncronas y asíncronas propuestas. Dos maneras de prepararnos ante eventuales contingencias como la presente son: 1) utilizar modelos de desarrollo instruccional (como ADDIE) que sean lo suficientemente flexibles como para introducir cambios, en cualquier momento, debidos a las diferencias de las modalidades presenciales y a distancia, y 2) analizar de manera precisa cuáles son las semejanzas y diferencias entre los modelos presenciales y remotos, ejercitarse en la medida de lo posible en ambas modalidades y generar herramientas adecuadas para transitar de una a otra en cualquier momento. 
Para los pedagogos, queda la tarea de explorar caminos como el presentado aquí o generar otras propuestas que contribuyan a enfrentar, de mejor manera, el mundo cambiante en el que vivimos.

Existen muchos otros factores en juego que no pude incluir en este artículo, pero que también es importante considerar y que deben discutirse en los diferentes contextos en donde sean pertinentes. Entre ellos se encuentran las planeaciones a nivel institucional que tienden a distinguir tajantemente entre los entornos de enseñanza presencial y remota. La pandemia ha mostrado que es tiempo de reflexionar sobre los modelos de enseñanza en general y la manera en que deben evolucionar. Para quienes no habían considerado seriamente incluir herramientas tecnológicas en sus propuestas metodológicas de enseñanza-aprendizaje, surge un fuerte llamado en el sentido de empezar a hacerlo con urgencia si es que desean sobrevivir. Esto no significa sacrificar la enseñanza en entornos presenciales, sin embargo, también hace falta reflexionar profundamente sobre cuál es el valor de la enseñanza presencial y cómo debe defenderse en un mundo que parece moverse cada vez más rápidamente (en el momento presente obligado por la situación de la pandemia), a favor de la digitalización y la enseñanza a distancia.

Considero importante también discutir cuáles son -o deben- ser las diferencias relevantes entre los modelos de enseñanza presencial y los de enseñanza remota. Está muy extendido el prejuicio de pensar que los modelos presenciales favorecen una enseñanza más «tradicional», en donde el docente transmite su conocimiento a un estudiante pasivo, mientras que se piensa que en los modelos de educación a distancia el estudiante es el protagonista de su propio aprendizaje. Sin embargo, ambas visiones son equivocadas y en realidad los dos modelos tienen ventajas y desventajas, las cuales variarán mucho dependiendo de todos los elementos que se presenten en el contexto de cada clase. En general, debe repensarse con mucha mayor profundidad qué es la labor educativa, qué debería ser y cómo podría llevarse a cabo, tanto en contextos «normales» o «tradicionales», como en otros extraordinarios y «de emergencia» como el de la pandemia.

La situación extraordinaria generada por ella, muestra la importancia de recurrir a la pedagogía como disciplina que puede ayudar a comprender las dificultades enfrentadas en los diferentes tipos de procesos de enseñanza-aprendizaje, y ofrecer herramientas útiles para lograr los objetivos propuestos en cada curso. Por otra parte, es responsabilidad de los docentes capacitarse adecuadamente, no solo en conocer su disciplina con profundidad, sino también en las diferentes herramientas para diseñar, planear y ejecutar sus cursos, así como en el conocimiento y uso de los diversos recursos tecnológicos que se generan constantemente. Finalmente también es esencial resaltar el papel que las autoridades institucionales tienen en sus manos: son las encargadas de contar con una visión clara de lo que significa la educación escolarizada, cuáles son sus objetivos y metodologías, y deben ofrecer - tanto a docentes como a estudiantes- las mejores condiciones para desarrollar su trabajo, desde el espacio físico en el que se realizarán las clases (en los entornos presenciales), como las herramientas y la capacitación necesaria para lograr los objetivos propuestos (tanto de modelos presenciales como a distancia).

La pandemia generó un reto mayor, pues se trató de una situación para la que no estábamos preparados; sin embargo, a unos meses de que inició, considero que a pesar de que podamos seguir entendiendo los cursos que aparecen en este contexto como cursos donde prima la «enseñanza remota de emergencia», deberíamos ir un paso más allá y dejar de considerar de «emergencia» una situación que se desarrollará - por lo que se 
vislumbra- uno o dos años al menos. Tanto los docentes como los directivos de las instituciones, tenemos herramientas al alcance para generar propuestas pedagógicas para enfrentar la incertidumbre descrita en este artículo, de modo que se ofrezcan procesos de enseñanza-aprendizaje, presenciales o a distancia, de muy buena calidad.

\section{REFERENCIAS}

«APOLO 21» ACOMPAÑA A DOCENTES EN DESARROLLO ONLINE. En https:// www.up.edu.mx/es/noticias/37869/apolo-21-acompana-docentes-en-desarrollo-online Fecha de consulta: 17-09-2020.

ACUERDO número 02 / 03/20 por el que se suspenden las clases en las escuelas de educación preescolar, primaria, secundaria, normal y demás para la formación de maestros de educación básica del Sistema Educativo Nacional, así como aquellas de los tipos medio superior y superior dependientes de la Secretaría de Educación Pública. En https: / / www.dof.gob.mx/nota detalle.php?codigo $=5589479 \& f e c h a=16 / 03 / 2020$ Fecha de consulta: 17-09-2020.

Becerril, C., Sosa, G., Delgadillo, M. y Torres, S. (2015). «Competencias básicas de un docente virtual». Revista de sistemas y gestión educativa, 2(4), septiembre de 2015, 882-887.

Belloch, C. (2011). Diseño instruccional. Unidad de Tecnología Educativa (UTE). Universidad de Valencia.

Chiappe-Laverde, A. (2008). «Diseño instruccional: oficio, fase y proceso». Educación y Educadores, 11(2), 229-239. En https:/ / www.redalyc.org/articulo.oa?id=83411215

Durán Rodríguez, R. A. (2015). La Educación Virtual Universitaria como medio para mejorar las competencias genéricas y los aprendizajes a través de buenas prácticas docentes. Tesis doctoral, Universidad Politécnica de Cataluña.

Esquivel Gámez, I. (Coord.) (2014). Los Modelos Tecno-Educativos, revolucionando el aprendizaje del siglo XXI. México.

Galindo, D. García, L., García, R., González, P., Hernández, P.C., López, M., Luna, V. y Moreno, C.I. (2020). «Recomendaciones didácticas para adaptarse a la enseñanza remota de emergencia». Revista Digital Universitaria (RDU), 21(5), septiembre-octubre, 2020. http:/ / doi.org/10.22201/ cuaieed.16076079e.2020.21.5.15

Gallego Arrufat, M.J. (2007). «Las fuentes docentes presenciales y virtuales del profesorado universitario». Teoría de la Educación. Educación y Cultura en la Sociedad de la Información. En https: / / www.redalyc.org/articulo.oa?id=201017334009

Góngora Parra, Y. y Martínez Leyet, O.L. (2012). «Del diseño instruccional al diseño de aprendizaje con aplicación de las tecnologías». Teoría de la educación. Educación y cultura en la sociedad de la información, TESI, 13(3), 342-360.

Goodyear, P., Salmon, G., Spector, J. M., Steeples, C. \& Tickner, S. (2001). «Competences for online teaching: A special report». Educational Technology Research and Development, 49(1), pp. 65-72. En https:// doi.org/10.1007/BF02504508 
Hodges, C., Moore, S., Lockee, B., Trust, T. \& Bond, A. (2020). «The Di-erence Between Emergency Remote Teaching and Online Learning». Educause Review. In https:// er.educause.edu/articles/2020/3/the-difference-between-emergency-remote-teaching-and-online-learning. Fecha de consulta: 17-09-2020.

Jandrić et al. (2020). «Teaching in the Age of covid 19». Postdigital Science and Education. En https: / / doi.org/10.1007/s42438-020-00169-6

Jardines Garza, F.J. (2011). «Revisión de los principales modelos de diseño instruccional». InnOvaciOnes de NegOciOs, 8(16), 357-389.

Londoño Giraldo E.P. (2011). «El diseño instruccional en la educación virtual: más allá de la presentación de contenidos». Revista Educación y Desarrollo Social, 6(2), julio-diciembre 2011, 112-127.

Morales-González, B., Edel-Navarro, R. y Aguirre-Aguilar, G. (2014). «Modelo ADDIE (análisis, diseño, desarrollo, implementación y evaluación): Su aplicación en ambientes educativos». En Esquivel Gámez, I. (Coord.) (2014). Los Modelos Tecno-Educativos, revolucionando el aprendizaje del siglo XXI. México.

Sotelo C., M.A.S., García, J.J.V., López, R.I.G. y Hernández, L.F.B. (2017). «Características del buen profesor de modalidad presencial y virtual desde la perspectiva de los estudiantes». European Scientific Journal, ESJ, 13(13). http://dx.doi.org/10.19044/esj.2017. $\underline{\mathrm{v} 13 \mathrm{n} 13 \mathrm{p} 78}$

UNAM a distancia. https: / / cuaieed.unam.mx/ Fecha de consulta: 17-09-2020. 